Pathophysiology Haemostasis and Thrombosis

\title{
Drug Based Approaches for Targeting Tumor Vasculature: Development of Combretastatin A4 Phosphate
}

\author{
David J. Chaplin \\ Oxigene Inc, Watertown, Mass., USA
}

Tumor vasculature represents an appealing target for the development of new cancer treatments. Most of the research effort has been focused on antiangiogenic therapy. However, recently there has been an increased interest in developing agents, which irreversibly damage the already formed neovasculature in tumors so called vascular targeting agents (VTAs).

VTAs differ from antiangiogenic agents in that, rather than preventing new blood vessel formation, they are designed to cause a rapid and selective vascular shutdown in tumors, which occurs over a period of minutes to hours. Moreover such agents are expected to be used in intermittent doses, to synergise with conventional treatments, rather than chronically over months or years. VTAs divide into two main classes (1) the biologics, which utilize antibodies and peptides to deliver toxins, procoagulant and proapoptotic effectors to tumor endothelium and (2) the small molecules, which do not specifically localize to tumor endothelium but exploit the known differences between tumor and normal endothelial cells to induce selective vascular dysfunction.

The approach has several advantages

- Each vessel provides the nutrition for and facilitates removal of waste products of metabolism from many thousands of tumor cells
- Since a vessel is much like any channel involved in transportation, it only has to be damaged at one point to block both upstream and downstream function

- It is not necessary to kill endothelial cells, a change of shape or function could also be effective

- The endothelial cell is adjacent to the blood stream therefore delivery problems of any therapeutic are minimized

- The target cell is a normal diploid cell, therefore drug resistant mutants are more unlikely to emerge

- A surrogate marker of biological activity i.e. blood flow, is readily measurable in the clinic

One of the most effective group of small molecule VTAs identified to date are certain tubulin depolymerizing agents the most studied compound being Combretastatin A4 phosphate (CA4P) which has completed Phase I trials and moved into Phase II trials. CA4P has been demonstrated to induce rapid, selective and extensive blood flow reductions in a large number of experimental tumor systems. These effects have been observed in spontaneous as well as transplanted tumor models. More recently clinical studies have demonstrated that tumor blood flow reductions are also seen in humans following administration of CA4P.

Although the mechanisms responsible for these effects have not been completely defined it has been shown that CA4P induces rapid shape changes in proliferating

David J. Chaplin

Head R\&D, Oxigene Inc

321 Arsenal St., Watertown, MA 02472 (USA)

Tel. +1617673 7811, Fax +16179249229

E-Mail dchaplin@oxigene.com

-

(1)


endothelial cells in culture. The fact that these changes occur over a similar time course to that seen with blood flow effects in vivo and that quiescent endothelial cells are much more resistant to such changes provides further evidence that this process is a key component in its action. These selective effects on cell shape reflect the critical role the tubulin cytoskeleton plays in maintenance of the elongated shape of endothelial cells when they are newly formed.

Whilst CA4P induces extensive necrosis in experimental tumors regressions are rarely seen, this is due to regrowth from a remaining viable rim of tumor cells dependant on the vessels in the adjacent normal tissues. These cells due to their location and proliferation rate are expected to be most sensitive to conventional treatments. Combination of CA4P with radiation and chemotherapy has been shown to enhance tumor response in experimental systems. Based on these findings and the clinical results to date Phase $\mathrm{Ib} / \mathrm{II}$ studies are now ongoing.

In parallel with the continued clinical development of CA4P in oncology, the search for 'second generation' vascular targeting agents which posses potent single agent activity is continuing. Our recent studies have identified
Oxi4503 as a compound which can induce tumor regression in a number of xenografted tumors. Phase I clinical trials with Oxi4503 are now planned.

In addition to cancer there are several other disease pathologies where abnormal neovascularization is an integral part of disease progression these including ocular disorders such as macular degeneration and retinopathy, vascular targeting approaches potentially offer a way of reversing the disease pathology by rendering nonfunctional the newly formed vasculature. The results obtained with CA4P in an experimental model of ocular neovascularization indicate it can not only stop but also reverse the process of neovascularization. Further studies evaluating the potential of CA4P in pathologies outside of oncology are now ongoing.

References

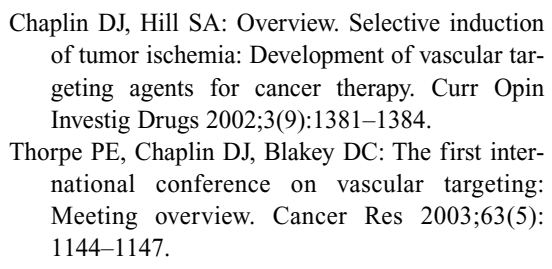
of tumor ischemia: Development of vascular targeting agents for cancer therapy. Curr Opin Investig Drugs 2002;3(9):1381-1384.

Thorpe PE, Chaplin DJ, Blakey DC: The first international conference on vascular targeting: Meeting overview. Cancer Res 2003;63(5): 1144-1147. 